PROCEEDINGS OF THE

AMERICAN MATHEMATICAL SOCIETY

Volume 138, Number 5, May 2010, Pages 1607-1619

S 0002-9939(10)10252-4

Article electronically published on January 13, 2010

\title{
NILPOTENT IDEALS IN POLYNOMIAL AND POWER SERIES RINGS
}

\author{
VICTOR CAMILLO, CHAN YONG HONG, NAM KYUN KIM, YANG LEE, \\ AND PACE P. NIELSEN
}

(Communicated by Birge Huisgen-Zimmermann)

\begin{abstract}
Given a ring $R$ and polynomials $f(x), g(x) \in R[x]$ satisfying $f(x) R g(x)=0$, we prove that the ideal generated by products of the coefficients of $f(x)$ and $g(x)$ is nilpotent. This result is generalized, and many well known facts, along with new ones, concerning nilpotent polynomials and power series are obtained. We also classify which of the standard nilpotence properties on ideals pass to polynomial rings or from ideals in polynomial rings to ideals of coefficients in base rings. In particular, we prove that if $I \leq R[x]$ is a left $T$-nilpotent ideal, then the ideal formed by the coefficients of polynomials in $I$ is also left $T$-nilpotent.
\end{abstract}

\section{INTRODUCTION}

Throughout this paper, all rings are associative rings with 1. It is well known that a polynomial $f(x)$ over a commutative ring is nilpotent if and only if each coefficient of $f(x)$ is nilpotent. But this result is not true in general for noncommutative rings. For example, let $R=\mathbb{M}_{n}(k)$, the $n \times n$ full matrix ring over some ring $k \neq 0$. Consider the polynomial $f(x)=e_{12}+\left(e_{11}-e_{22}\right) x-e_{21} x^{2}$, where the $e_{i j}$ 's are the matrix units. In this case $f(x)^{2}=0$, but $e_{11}-e_{22}$ is not nilpotent. (In fact, when $n=2$ this is a unit.) The trouble seems to arise from the fact that if $f(x)$ is nilpotent, then so is the ideal $\langle f\rangle$ over a commutative ring, but not necessarily over a noncommutative ring. Indeed, for a general ring $R, f(x) R[x]$ is a nilpotent right ideal of $R[x]$ if and only if $A_{f} R$ is a nilpotent right ideal of $R$, where $A_{f}$ is the set of coefficients of $f(x)$. We provide some extensions and generalizations of this result.

Fields [1, Corollary 1] proved that if $R$ is a commutative Noetherian ring, then a power series $g(x)$ over $R$ is nilpotent if and only if each coefficient of $g(x)$ is nilpotent. He also provided an example showing that the condition " $R$ is Noetherian" is not redundant [1, Example 1]. On the other hand, when $R$ is a general ring, $X$ is a set of noncommuting indeterminates with $|X| \geq 2$, and $I$ is a right ideal of $R \llbracket X \rrbracket$, it is known that if $I$ is nil of bounded index, then the ideal generated by the coefficients of the power series in $I$ is nilpotent of the same index [9, Theorem 2]. The case for power series in one variable is much more difficult, and we refer the reader to [10]

Received by the editors June 9, 2009, and, in revised form, September 15, 2009.

2000 Mathematics Subject Classification. Primary 16N40; Secondary 16U80.

Key words and phrases. Bounded index of nilpotence, left $T$-nilpotent, locally nilpotent, nil, nilpotent, polynomial ring, power series ring. 
for more information. In this paper we prove a generalization of the fact that if we have a power series $f(x) \in R \llbracket x \rrbracket$, where $A_{f} R$ is finitely generated, then $f(x) R \llbracket x \rrbracket$ is nilpotent if and only if $A_{f} R$ is nilpotent. The methods we employ work for power series over any number of noncommuting indeterminates, without invoking hypotheses concerning ideals in such rings. Throughout the paper, we let $A_{f}$ be the set in $R$ consisting of all the "coefficients" of $f(x)$ whether $f(x)$ be a polynomial, power series, or something more exotic.

In the last few sections we collect much of the folklore concerning the behavior of nilpotence properties on (one-sided) ideals passing to polynomial extensions, or from polynomials down to the base ring. It appears that both Example 15 and Theorem 18 are new. For more information about the behavior of radical properties under polynomial extensions, we refer the reader to the recent book 3 .

\section{Generating Nilpotent ideals}

As mentioned in the introduction, if $R$ is a noncommutative ring and $f(x) \in R[x]$ is nilpotent, this does not imply that the coefficients of $f(x)$ are nilpotent. However, if we assume a small "commutativity-like" hypothesis, we obtain stronger conditions on the coefficients of $f(x)$.

Theorem 1. Let $R$ be a ring, and let $f(x)=\sum_{i=0}^{m} a_{i} x^{i}$ and $g(x)=\sum_{j=0}^{n} b_{j} x^{j}$ be polynomials in $R[x]$. If $f(x) R g(x) R=0$, then $a_{i} R b_{j} R$ is a nilpotent right ideal, for each pair $(i, j)$.

Proof. We work by induction on $k=i+j$. If $k=0$, then $i=j=0$. We know $a_{0} R b_{0} R=0$ by looking at the degree zero term in $f(x) R g(x) R=0$. Thus $a_{0} R b_{0} R$ is trivially nilpotent.

So, we may assume $k \geq 1$ and that the claim is true for all smaller values by inductive assumption. From the degree $k$ coefficient in $f(x) R g(x) R=0$ we obtain

$$
\sum_{t=0}^{k} a_{t} r b_{k-t} s=0
$$

for arbitrary $r, s \in R$. In particular, upon specializing $r$ in equation (11) to $u b_{0} v$ (with $u, v \in R$ arbitrary) we have $\sum_{t=0}^{k} a_{t} u b_{0} v b_{k-t} s=0$. But then

$$
a_{k} u b_{0} v b_{0} s=-\sum_{t=0}^{k-1} a_{t} u b_{0} v b_{k-t} s \in \sum_{t<k} a_{t} R b_{0} R .
$$

The right ideal on the right hand side is nilpotent (since it is a finite sum of such, by inductive hypothesis). Thus, $\left(a_{k} R b_{0} R\right)^{2} \subset a_{k} R b_{0} R b_{0} R$ is a nilpotent right ideal, and hence so is $a_{k} R b_{0} R$.

Repeat the argument in the previous paragraph by specializing $r$ to $u b_{j} v$ instead of $u b_{0} v$, and induct on $j$. Note that the previous paragraph consists of checking the base case. In this more general case, equation (11) then yields

$$
a_{k-j} u b_{j} v b_{j} s=-\left(\sum_{t=0}^{k-j-1} a_{t} u b_{j} v b_{k-t} s\right)-\left(\sum_{t=k-j+1}^{k} a_{t} u b_{j} v b_{k-t} s\right) .
$$

In particular, $a_{k-1} R b_{j} R b_{j} R \subseteq\left(\sum_{t<k-j} a_{t} R b_{j} R\right)+\left(\sum_{i^{\prime}+j^{\prime}=k, j^{\prime}<j} a_{i^{\prime}} R b_{j^{\prime}} R\right)$, and this is nilpotent by the inductive assumptions on $k$ and $j$. By the same reasoning 
as in the previous paragraph, we obtain that $a_{k-j} R b_{j} R$ is nilpotent, finishing both inductions.

This theorem can be proven much more quickly by using an idea brought to our attention by the referee. Notice that over either of the two rings $R^{\prime}=R /\left\langle a_{m}\right\rangle$ and $R^{\prime \prime}=R /\left\langle b_{n}\right\rangle$, we have a reduced value of $k$. By the inductive hypothesis, the image of $T=a_{i} R b_{j} R$ is nilpotent in both of the quotient rings $R^{\prime}$ and $R^{\prime \prime}$. Thus, some power of $T$ lives in the product $\left\langle a_{m}\right\rangle \cdot\left\langle b_{n}\right\rangle$, which is nilpotent in $R$.

We retain the original proof, as it allows some generalizations not available to the faster proof, such as (we will see) to the case of power series rings. One should also note that the nilpotency index on $a_{i} R b_{j} R$ can easily be made effective in terms of the pair $(i, j)$ by modifying the inductive hypothesis employed in the initial proof. There are many other ways to generalize this theorem. The first is to notice that we can make it into an "if and only if" statement as follows:

Proposition 2. Let $R$ be a ring, and let $f(x)=\sum_{i=0}^{m} a_{i} x^{i}, g(x)=\sum_{j=0}^{n} b_{j} x^{j} \in$ $R[x]$. The set $f(x) R g(x) R$ is nilpotent if and only if $a_{i} R b_{j} R$ is a nilpotent right ideal for each pair $(i, j)$.

Proof. The forward direction is a simple mutatis mutandis argument using the first proof. The backward direction is a consequence of the fact that there are only finitely many pairs $(i, j)$.

Note that since $x \in R[x]$ is central we have that the set $f(x) R g(x) R$ is nilpotent if and only if the right ideal $f(x) R[x] g(x) R[x]$ is nilpotent. In other situations we will consider later, we don't have a similar "centrality" condition, which is why we stated the theorem as we did. However, using this information we do obtain the following classical results.

Corollary 3. Let $R$ be a ring. The right ideal $f(x) R[x]$ of $R[x]$ is nilpotent if and only if the right ideal $A_{f} R$ of $R$ is nilpotent.

Denote the sum of the nilpotent ideals of $R$, called the Wedderburn radical, by $W(R)$.

Corollary 4. For any ring $R, W(R[x])=W(R)[x]$.

Another way to generalize Theorem 1 is to consider the product of more than two polynomials. We note that since our rings are possibly noncommutative, when we write a product $\prod_{i=1}^{n} a_{i}$ it signifies the left-to-right product $a_{1} a_{2} \cdots a_{n}$.

Proposition 5. Let $n \in \mathbb{Z}_{>0}$, let $R$ be a ring, and for each $1 \leq \ell \leq n$ let $f_{\ell}(x)=$ $\sum_{i=0}^{n_{\ell}} a_{i, \ell} x^{i} \in R[x]$. The set $\prod_{\ell=1}^{n}\left(f_{\ell}(x) R\right)$ is nilpotent if and only if $\prod_{\ell=1}^{n}\left(a_{i_{\ell}, \ell} R\right)$ is a nilpotent right ideal, for all choices $0 \leq i_{\ell} \leq n_{\ell}$ (for each $\ell$ ).

Proof. As before, the reverse implication is easy. For the forward implication, we simplify to the case $\prod_{\ell=1}^{n}\left(f_{\ell}(x) R\right)=0$, as the more general case follows from this case but requires more extensive notation. Let $S=\left\{\left(i_{1}, i_{2}, \ldots, i_{n}\right) \mid 0 \leq i_{\ell} \leq n_{\ell}\right\}$ be the set of $n$-tuples whose entries consist of indices for the coefficients of the polynomials. We can order $S$ by saying

$$
\left(i_{1}, i_{2}, \ldots, i_{n}\right)<\left(j_{1}, j_{2}, \ldots, j_{n}\right)
$$

if and only if there exists an integer $m$ in the range $1 \leq m \leq n$ so that $\sum_{\ell=m^{\prime}}^{n} i_{\ell}=$ $\sum_{\ell=m^{\prime}}^{n} j_{\ell}$ when $1 \leq m^{\prime}<m$ but $\sum_{\ell=m}^{n} i_{\ell}<\sum_{\ell=m}^{n} j_{\ell}$. Given an $n$-tuple $s=$ $\left(i_{1}, i_{2}, \ldots, i_{n}\right) \in S$, let $B_{s}=\prod_{\ell=1}^{n}\left(a_{i_{\ell}, \ell} R\right)$. 
We work by induction on the well-ordered set $S$ to prove that $B_{s}$ is nilpotent for each $s \in S$. The claim is clearly true for the $n$-tuple $s_{0}=(0,0, \ldots, 0)$. Fix $s=\left(i_{1}, i_{2}, \ldots, i_{n}\right) \in S$, and suppose by induction that the claim is true for all $n$-tuples $t<s$. Set $k=\sum_{\ell=1}^{n} i_{\ell}$. Looking at the degree $k$ coefficient in the equation $\prod_{\ell=1}^{n}\left(f_{\ell}(x) R\right)=0$ yields

$$
\sum_{j_{1}+j_{2}+\cdots+j_{n}=k}\left(\prod_{\ell=1}^{n}\left(a_{j_{\ell}, \ell} r_{\ell}\right)\right)=0
$$

with $r_{\ell} \in R$ arbitrary. If we specialize each $r_{\ell}$ to $s_{\ell, \ell} \prod_{\ell^{\prime}=\ell+1}^{n}\left(a_{i_{\ell^{\prime}, \ell^{\prime}}} s_{\ell^{\prime}, \ell}\right)$ (for arbitrary $s_{\ell^{\prime}, \ell} \in R$, for all $\ell^{\prime}>\ell$ ), then just as in the proof of Theorem 11 equation (2) implies that $C_{s}=\prod_{\ell=1}^{n}\left(\prod_{\ell^{\prime}=\ell}^{n} a_{i_{\ell^{\prime}}, \ell^{\prime}} R\right)$ belongs to $\sum_{t<s} B_{t}$, which is nilpotent. On the other hand $B_{s}^{n} \subseteq C_{s}$, and so $B_{s}$ is similarly nilpotent. This completes the induction.

To generalize our theorem another way, note that the induction on $k$ in Theorem 1 does not rely in any way on the fact that $k$ is bounded by $m+n$. In other words, the same proof works for power series rings by merely replacing $m$ and $n$ by $\infty$.

Proposition 6. Let $n \in \mathbb{Z}_{>0}$, let $R$ be a ring, and for each $1 \leq \ell \leq n$ set $f_{\ell}=$ $\sum_{i=0}^{\infty} a_{i, \ell} x^{i} \in R \llbracket x \rrbracket$. If $\prod_{\ell=1}^{n}\left(f_{\ell}(x) R\right)$ is nilpotent, then $\prod_{\ell=1}^{n}\left(a_{i_{\ell}, \ell} R\right)$ is a nilpotent right ideal, for all choices $0 \leq i_{\ell}$ (for each $\ell$ ).

Corollary 7. Let $R$ be a ring and $f(x) \in R \llbracket x \rrbracket$. Suppose $A_{f} R$ is finitely generated. The right ideal $f(x) R \llbracket x \rrbracket$ is nilpotent if and only if $A_{f} R$ is nilpotent.

The proof of Theorem 1 does not rely on the fact that we are working with polynomials, except when we claim the ability to order the products $a_{i} R b_{j} R$ in "compatible" ways. This point is emphasized in the proof of Propositions 5 and 6 , More generally, we can work in the context of monoid rings.

Proposition 8. Let $n \in \mathbb{Z}_{>0}$, let $R$ be a ring, and let $G$ be a monoid so that each finitely generated submonoid is an ordered monoid. Let $\left\{g_{1}, g_{2}, \ldots, g_{u}\right\} \subseteq G$. For each $1 \leq \ell \leq n$, set $f_{\ell}=\sum_{i=1}^{u} a_{i, \ell} g_{i} \in R[G]$. If $\prod_{\ell=1}^{n}\left(f_{\ell} R\right)=0$, then $\prod_{\ell=1}^{n}\left(a_{i_{\ell}, \ell} R\right)$ is a nilpotent right ideal, for all choices $1 \leq i_{\ell} \leq u$ (for each $\ell$ ).

Proof. One follows the proof of Proposition 5, but since some of the needed changes are not obvious we clarify them here. First, fix an ordering on the submodule of $G$ generated by $\left\{g_{1}, \ldots, g_{u}\right\}$. Second, define an ordering on $n$-tuples of indices by the rule:

$$
\left(i_{1}, i_{2}, \ldots, i_{n}\right)<\left(j_{1}, j_{2}, \ldots, j_{n}\right)
$$

if and only if there exists an integer $m$ in the range $1 \leq m \leq n$ so that $\prod_{\ell=m^{\prime}}^{n} g_{i_{\ell}}=$ $\prod_{\ell=m^{\prime}}^{n} g_{j_{\ell}}$ when $1 \leq m^{\prime}<m$ but $\prod_{\ell=m}^{n} g_{i_{\ell}}<\prod_{\ell=m}^{n} g_{j_{\ell}}$. One works by induction, as before, on the set $S$ of these $n$-tuples under this well-ordering.

The third major change is that equation (2) should be modified to include only those terms in the product $f_{1} R f_{2} R \cdots f_{n} R=0$ with common support $g \in G$ (for some given element $g$ ). The rest of the proof involves only minor changes.

Corollary 9. Let $R$ be a ring, let $X$ be a set of indeterminates, and let $f, g \in R\langle X\rangle$ be elements of the free ring in noncommuting indeterminates over $X$. We can write $f=\sum_{i \in I} a_{i} w_{i}$ and $g=\sum_{i \in I} b_{i} w_{i}$, where $I$ is a finite set, $a_{i}, b_{i} \in R$ for all $i \in I$, and $\left\{w_{i}\right\}_{i \in I}$ is a subset (without repetitions) of the free monoid generated by $X$. 


\section{If $f R g R=0$, then $a_{i} R b_{j} R$ is a nilpotent right ideal for each pair $(i, j)$.}

In the context of the previous corollary, if $|X| \geq 2$ and we assume the stronger condition that $f R\langle X\rangle g=0$, then one can easily prove that $a_{i} R b_{j}=0$.

Recall that if $\sigma: R \rightarrow R$ is a ring homomorphism, we can define the skewpolynomial ring $R[x ; \sigma]$ which, as an underlying set, is the same as the polynomial ring but has the twisted multiplication

$$
\left(\sum_{i=0}^{m} a_{i} x^{i}\right)\left(\sum_{j=0}^{n} b_{j} x^{j}\right)=\sum_{k=0}^{m+n}\left(\sum_{i+j=k} a_{i} \sigma^{i}\left(b_{j}\right)\right) x^{k} .
$$

Once again, the same proof as for Theorem 1 with only small changes yields:

Proposition 10. Let $R$ be a ring, and let $\sigma$ be a ring epimorphism on $R$. Let $f(x)=\sum_{i=0}^{m} a_{i} x^{i}$ and $g(x)=\sum_{j=0}^{n} b_{j} x^{j} \in R[x ; \sigma]$. If $f(x) R g(x) R=0$, then $a_{i} R \sigma^{i}\left(b_{j}\right) R$ is a nilpotent right ideal, for each pair $(i, j)$.

Note that these last few results can be appropriately extended to arbitrary finite products, or we may replace the condition $f(x) R g(x) R=0$ with the hypothesis that $f(x) R g(x) R$ is nilpotent, exactly as when we extended Theorem 1 to Propositions 2 and 5 (with the usual mutatis mutandis caveat).

\section{Nilpotence PROPERTIES}

Let $R$ be a ring. Following [6], we let $\operatorname{rad}(R), \mathrm{Nil}^{*}(R), \mathrm{L}-\mathrm{rad}(R)$, and $\mathrm{Nil}_{*}(R)$ denote, respectively, the Jacobson radical (the intersection of all maximal left ideals), the upper nilradical (the sum of all nil ideals), the Levitsky radical (the sum of all locally nilpotent ideals), and the lower nilradical (the intersection of all prime ideals). One has the containments $W(R) \subseteq \mathrm{Nil}_{*}(R) \subseteq \mathrm{L}-\operatorname{rad}(R) \subseteq \mathrm{Nil}^{*}(R) \subseteq \operatorname{rad}(R)$, where each containment may be proper. More generally, let $I$ be a right ideal in $R$. The following implications hold for some common nilpotence conditions on $I$ :

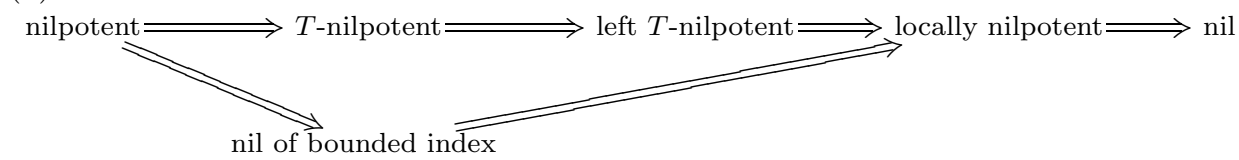

We refer the reader to [6] for basic information about these properties and proofs of all of these implications. When we say that $I$ is $T$-nilpotent we mean that $I$ is both left and right $T$-nilpotent. We note that there are no other implications (except those implied by composition) even if $I$ is finitely generated as a right ideal due to the following examples.

Example 11. (1) Let $R=\mathbb{Q}\left\langle x_{1}, x_{2}, \ldots\right\rangle$ with relations $x_{1}^{2}=0, x_{i} x_{j}=0$ when $i, j>1$, and $x_{m} x_{1} x_{n}=0$ whenever $n \geq m$. The right ideal $I=x_{1} R$ is left $T$-nilpotent but not right $T$-nilpotent and not nil of bounded index since $y_{n}=$ $x_{1} x_{n+1}+x_{1} x_{n}+\cdots+x_{1} x_{2}$ has nilpotency index $n$. (Note: We can similarly choose the relations so that $I=x_{1} R$ is right $T$-nilpotent but not left $T$-nilpotent and not nil of bounded index.)

(2) Let $R=\mathbb{Q}\left\langle x_{1}, x_{2}, \ldots\right\rangle$ with relations $x_{1}^{2}=0, x_{i} x_{j}=0$ when $i, j>1$, $x_{i} x_{1} x_{j}=x_{j} x_{1} x_{i}$ when $i, j>1$, and $x_{m} \prod_{i=1}^{m}\left(x_{1} x_{\ell_{i}}\right)=0$ for any choice of indices $\ell_{i}$. The right ideal $I=x_{1} R$ is left and right $T$-nilpotent but not nil of bounded index. 
(3) Let $R=\mathbb{F}_{2}\left\langle x_{1}, x_{2}, \ldots\right\rangle$ subject to the relations $x_{1}^{2}=0, x_{i} x_{j}=0$ when $i, j>1, x_{i} x_{1} x_{i}=0$ for all $i>1$, and $x_{i} x_{1} x_{j}=x_{j} x_{1} x_{i}$ when $i, j>1$. The right ideal $I=x_{1} R$ has bounded index of nilpotency 3 but is neither left nor right $T$-nilpotent. We note that an ideal which has bounded index of nilpotency 2 is automatically nilpotent, so this example is optimal.

(4) Local nilpotence is decidable on finite subsets. Any left or right $T$-nilpotent right ideal lies in $\operatorname{Nil}_{*}(R)$ by [6. Proposition 23.5], and similarly any right ideal which is nil of bounded index lies in $\operatorname{Nil}_{*}(R)$ by [5]. So the nonexistence of all other implications boils down to the possible strict inclusions $\operatorname{Nil}_{*}(R) \subset \operatorname{L-rad}(R) \subset$ $\operatorname{Nil}^{*}(R)$. An example of the first inclusion being strict is constructed in [6, p. 167]. The first example of the other inclusion being strict arose from Golod's work in the 1960's. We refer the reader to [12] for a stronger example, which shows that the inclusion can be strict even for polynomial rings.

Remark 1 . The previous examples must all be noncommutative due to the following facts: (1) in a commutative ring any nil right ideal is locally nilpotent, and (2) in a commutative ring, any finitely generated nil right ideal is nilpotent.

Remark 2. There are no further implications in diagram (3) when $I$ is a two-sided ideal by similar constructions to those above. We leave it to the reader to construct examples.

For a thorough treatment of the behavior of certain radical properties under polynomial extensions, we direct the reader to Chapter 4 of [3]. We compile here some results which are scattered across the literature. A finite sum of right ideals which are nil of bounded index is still of bounded index by [5, Theorem 3]. Following [5] we define

$$
N(R)=\{a \in R \mid a R \text { is nil of bounded index }\}
$$

and we have $W(R) \subseteq N(R) \subseteq \mathrm{Nil}_{*}(R)$. One can define the higher Wedderburn radicals by setting $W_{1}(R)=W(R), W_{\alpha+1}(R)=\left\{a \in R \mid a R+W_{\alpha}(R) \in W\left(R / W_{\alpha}\right)\right\}$, and, if $\alpha$ is a limit ordinal, $W_{\alpha}(R)=\bigcup_{\beta<\alpha} W_{\beta}(R)$. It is an easily deduced fact that these stabilize to the lower nilradical [6, Exercise 10.11]. Further, from our work in the previous section we know that $W_{\alpha}(R[x])=W_{\alpha}(R)[x]$, and hence we have another proof of Amitsur's theorem stating that $\mathrm{Nil}_{*}(R[x])=\mathrm{Nil}_{*}(R)[x]$; see [6. Theorem 10.19]. Note that Klein proved in [5] that $W_{1}(R) \subseteq N(R) \subseteq W_{2}(R)$, where either containment may be proper or an equality.

One may ask how the properties in diagram (3) behave when passing to polynomial rings or from polynomial rings down to the base ring. We will completely answer both of these questions in the remainder of this section. Most of the implications are classical, and our main contributions are Example 15 and Theorem 18.

The following "Going up" theorem is a collection of results from the literature:

Theorem 12 (Going up). Let $\mathcal{P}$ be any property in diagram (3) except "nil". Let $R$ be a ring, and let $J$ be a right ideal in $R$. If $J$ has property $\mathcal{P}$, then $J[x]$ has property $\mathcal{P}$. No other implications going from $J$ to $J[x]$ exist among those properties listed in diagram (3), even if $J$ is an ideal, except those implied by transitivity.

Proof. The proofs of " $\mathcal{P}$ on $J$ implies $\mathcal{P}$ on $J[x]$ " when $\mathcal{P} \neq$ "nil" are either folklore or straightforward. Gardner proved in [2, Proposition 1.4] that if $R$ is a (nonunital) left $T$-nilpotent ring, then so is $R[x]$. The case where $\mathcal{P}=$ "nil of bounded index" was dealt with by Klein [4. Smoktunowicz and Puczyłowski [13] gave an example of 
a polynomial ring which is Jacobson radical but not upper nilradical, thus dealing with the case where $\mathcal{P}=$ "nil". All other non-implications are a trivial consequence of the non-implications in diagram (3) by considering constant polynomials.

Corollary 13. There are no extra implications in diagram (3), even when we restrict to ideals in polynomial rings.

Proof. Let $\mathcal{P}$ and $\mathcal{Q}$ be two properties of the diagram with $\mathcal{P} \nRightarrow \mathcal{Q}$. Fix some ideal $J \leq R$, for some ring $R$, so that $J$ has property $\mathcal{P}$ but not property $\mathcal{Q}$.

We first consider the case where $\mathcal{P} \neq$ "nil". By the previous theorem $J[x]$ has property $\mathcal{P}$. If $J[x]$ has property $\mathcal{Q}$, then so does $J$ by considering the constant polynomials in $J[x]$. But this would contradict our construction of $J$. Thus $J[x]$ does not have property $\mathcal{Q}$.

Now suppose $\mathcal{P}=$ "nil". By a result of Amitsur and McCoy, we have the equality $\mathrm{Nil}_{*}(R[x])=\mathrm{Nil}_{*}(R)[x]$, as proven in [6, Theorem 10.19]. It is also easy to check that the equality L-rad $(R[x])=\mathrm{L}-\operatorname{rad}(R)[x]$ always holds. (To prove this, first reduce to the case where L- $\operatorname{rad}(R)=0$, and then look at the nonzero coefficients in L-rad $(R[x])$ in the smallest possible degree.) Thus, the inclusion $\mathrm{Nil}_{*}(R[x]) \subset \mathrm{L}-\operatorname{rad}(R[x])$ is proper when the corresponding inclusion is proper over $R$.

Finally, by [12, fix a ring $R$ with $\operatorname{rad}(R[x, y])$ not nil. By another theorem of Amitsur we have $\operatorname{rad}(R[x, y]) \subseteq \operatorname{Nil}^{*}(R[x])[y]$; see [6. Theorem 5.10]. It cannot be the case that $\operatorname{Nil}^{*}(R[x])$ (which is a nil ideal) equals L-rad $(R[x]$ ), since (as we just saw) local nilpotence passes up to the polynomial ring.

The "Going down" theorem is more complicated than the "Going up" theorem.

Theorem 14 (Going down). Let $R$ be a ring, let $I$ be a right ideal in $R[x]$, and let $A_{I}$ be the right ideal of coefficients of polynomials in $I$. Let

$$
\mathcal{P} \in\{\text { "T-nilpotent", "left T-nilpotent", "locally nilpotent", "nil" }\} .
$$

If I has property $\mathcal{P}$, then $A_{I}$ has property $\mathcal{P}$. No other implications going from I to $A_{I}$ exist among those properties listed in diagram (3), even if $I$ is an ideal, except those implied by transitivity.

We prove this theorem in a number of steps. We begin with an example of a commutative ring $R$ with an ideal $L$ in $R[x]$ which is nilpotent (in fact $L^{2}=0$ ) but such that $A_{L}$ is not even nil of bounded index.

Example 15. We modify the example in [10, Theorem 11]. Let $F=K\left[s_{1}, s_{2}, \ldots\right]$ be the polynomial ring in a countably infinite set of variables, over a field $K$ of characteristic zero. Let $I$ be the ideal of $F$ generated by elements $h_{k}=\sum_{i=1}^{k-1} s_{i} s_{k-i}$, for $k \geq 2$. Let $H$ be the ideal of $F$ consisting of polynomials with zero constant term.

Fix an integer $m \geq 3$, and fix another integer $n>m^{m^{2}}$ (which depends on $m$ ). Let

$$
\left.J_{m}=\left\langle s_{i_{1}} \cdots s_{i_{t}}\right| t>m \text { or } i_{1}+\cdots+i_{t}>m^{m^{2}}\right\rangle
$$

which is an ideal of $F$, and put $I_{n}=\left\langle s_{\alpha} \mid \alpha>n\right\rangle$.

Let $R_{n}=F /\left(I+I_{n}\right)$. The ideal $H^{\prime}=H /\left(I+I_{n}\right)$ of $R_{n}$ is nilpotent, but we claim that the index of nilpotency is $>m$. The argument is as follows. Since $I_{n} \subseteq J_{m}$ it suffices to prove that $\left(H /\left(I+J_{m}\right)\right)^{m} \neq 0$. Hence, it suffices to prove 
that $\left(H^{m}+J_{m}\right) / J_{m} \nsubseteq \subseteq\left(I+J_{m}\right) / J_{m}$, which follows by dimension arguments as worked out in 10 .

We now make the dependence of $n$ on $m$ explicit by writing $n=n_{m}$. Set $R=\prod_{m \geq 3} R_{n_{m}}$, and for each $m \geq 3$ set $f_{m}(x)=\sum_{j=1}^{n_{m}} \overline{s_{j}} x^{j} \in R_{n_{m}}$. Note that we have $\left(f_{m}(x)\right)^{2}=0$ due to the relations in the ideal $I$. Finally form the ideal

$$
L=\left\langle\left(f_{3}(x), 0,0,0, \ldots\right),\left(0, f_{4}(x), 0,0, \ldots\right),\left(0,0, f_{5}(x), 0, \ldots\right), \ldots\right\rangle
$$

in $R[x]$. We have $L^{2}=0$, but $A_{L}$ is not nil of bounded index, since the index of nilpotency for the $m$ th coordinate is greater than $m$.

Corollary 3 demonstrates that if a single polynomial generates a nilpotent ideal, then so do the coefficients, and the same is true for any finitely generated ideal. On the other hand, the previous example shows that the result is not true, in general, for countably generated ideals. However, we can prove a slight extension of Corollary 3 by working with polynomials of bounded degree. For any right ideal $I$ of $R[x]$, we denote by $A_{I, n}$ the subset of $A_{I}$ generated by the coefficients of the polynomials in $I$ of degree $\leq n$.

Proposition 16. Let $R$ be a ring and $I$ a right ideal of $R[x]$. If $I^{n}=0$, then $\left(A_{I, m}\right)^{n^{m+1}}=0$ for each $m \geq 0$. Similarly, if $I$ is nil of bounded index, then so is $A_{I, m}$ for each $m \geq 0$. In particular, if $I$ is generated by polynomials of bounded degree, then $A_{I}$ is nilpotent (respectively, nil of bounded index) when I is.

Proof. Assume $I^{n}=0$. We first claim that $A_{I, s}^{n} \subseteq A_{I, s-1}$. To prove this claim let $\left\{f_{t}\right\}_{t \leq n}$ be an arbitrary, but finite, set of polynomials in $I$. Write $f_{t}(x)=$ $\sum_{i=1}^{n_{t}} a_{i, t} x^{i}$. It suffices to show that for $s \in \mathbb{Z}_{>0}$, an element $\alpha=a_{s, 1} a_{s, 2} \cdots a_{s, n}$ lies inside the right ideal of $R$ generated by $a_{i, t}$ for $i<s$. To prove this subclaim, compute the degree $s n$ coefficient of $f_{1}(x) f_{2}(x) \cdots f_{n}(x)=0$. One term equals $\alpha$ while all of the other terms live inside the right ideal of $R$ generated by $a_{i, t}$ for $i<s$. Since these terms all sum to zero, our claim is proven.

Note that $A_{I, 0}^{n}=0$. Since $A_{I, 1}^{n} \subseteq A_{I, 0}$ this means that $A_{I, 1}^{n^{2}}=0$. Repeating this argument, we get $A_{I, m}^{n^{m+1}}=0$ for each $m \geq 0$.

Now assume instead that $I$ is nil of bounded index $n$. First note that $A_{I, 0}$ is clearly nil of bounded index, with index at most $k_{0}=n$. Assume by induction that $A_{I, s-1}$ is nil of bounded index $k_{s-1}$. Taking $a \in A_{I, s}$, fix some polynomial $f(x) \in I$ with $a$ being the coefficient of $x^{s}$ in $f(x)$. Looking at the degree $s n$ term in $f(x)^{n}=0$ we see that $a^{n} \in A_{I, s-1}$ (using the same argument as above). Thus, $A_{I, s}$ is nil of bounded index, with index at most $k_{s-1}^{n}$. The rest of the proof follows easily.

Corollary 17. Let $R$ be a ring; then $N(R[x])=N(R)[x]$.

Proof. We have $N(R)[x] \subseteq N(R[x])$ by Theorem 12, On the other hand $A_{N(R[x])}=$ $\sum_{m \geq 0} A_{N(R[x]), m} \subseteq N(R)$ by the previous proposition, so $N(R[x]) \subseteq A_{N(R[x])}[x] \subseteq$ $N(R)[x]$

We now continue the proof of Theorem 14. First, if $I$ is nil, then $A_{I}$ is nil; this follows from the fact we mentioned earlier, and first proved by Amitsur, that $\operatorname{rad}(R[x]) \subseteq \mathrm{Nil}^{*}(R)[x]$. Second, as also already mentioned, we have the equality $\operatorname{L}-\operatorname{rad}(R[x])=\mathrm{L}-\operatorname{rad}(R)[x]$. Thus, if $I$ is locally nilpotent, then $A_{I}$ is locally nilpotent. 
The non-implications in Theorem 14 are all proved along similar lines. We give a single example of how this is done, and leave the other cases to the reader. Suppose, by way of contradiction, that $I$ being nil implies $A_{I}$ is locally nilpotent. By the "Going up" theorem this would mean $A_{I}[x] \supset I$ is locally nilpotent. But there is an ideal $I \leq R[x]$ which is nil but not locally nilpotent, since (as we previously proved) there are no extra implications in diagram (3) for polynomial rings. This is the desired contradiction.

All that remains for proving the "Going down" theorem is the following result:

Theorem 18. Let $R$ be a ring, let $I$ be a right ideal in $R[x]$, and let $A_{I}$ be the right ideal in $R$ of coefficients of polynomials in I. If I is left (respectively, right) $T$-nilpotent, then $A_{I}$ is left (respectively, right) T-nilpotent.

Proof. Assume $I$ is left $T$-nilpotent, and let $\alpha=\left(a_{1}, a_{2}, a_{3}, \ldots\right)$ be a sequence of elements in $A_{I}$. Fix polynomials $f_{i}(x) \in I$ so that $a_{i}$ is a coefficient of $f_{i}(x)$. Write $f_{i}(x)=\sum_{j=0}^{n_{i}} b_{i, j} x^{j}$ and let $B=\left\{b_{i, j} \mid \forall i, j\right\}$ be the set of all coefficients of the polynomials $\left\{f_{1}, f_{2}, \ldots\right\}$. We think of the polynomials $f_{1}, f_{2}, \ldots$ as being distinct (even if they, technically, are the same element in the ring $R[x]$ ) and each of their coefficients as being distinct. More formally, we could let $B$ be the set of ordered tuples $(b, i, j)$, where $b \in R$ is the coefficient of $f_{i}(x)$ at degree $j$. However, it is easier to just think of the subscripts on these coefficients as place-holders, so that we do not have to overly complicate the notation. We leave it to the reader to verify that this slight equivocation causes no problems in the proof.

By cardinality reasoning, we can easily construct an injective set map $\varphi: B \rightarrow$ $\mathbb{Z}_{>0}$ with the property that $\varphi\left(b_{i, j}\right)>i$. In effect, the map $\varphi$ will tell us how to multiply polynomials to take advantage of the fact that $I$ is left $T$-nilpotent, and in particular will give us a set of "reductions" that do not allow for looping. Let $B_{i}=\left\{b_{i, 0}, \ldots, b_{i, n_{i}}\right\}$ be the set of coefficients of $f_{i}(x)$ (again, thought of as being distinct from one another). Set $C_{1}=\{1\}$ and inductively put $C_{\ell}=\left\{\varphi\left(b_{i, k}\right) \mid i \in\right.$ $C_{\ell-1}, k$ arbitrary $\} \cup C_{\ell-1}$. We also let

$$
F_{\ell}=\left\{f_{i}(x) r_{i} \mid i \in C_{\ell}, r_{i} \in \prod_{j=i+1}^{\varphi\left(b_{i, k}\right)-1} B_{j}, \text { where } k \text { is arbitary }\right\} .
$$

Note that $C_{\ell}$ is always a finite set, and hence so is $F_{\ell}$, for each $\ell \geq 1$. By König's tree lemma and the fact that $I$ is left $T$-nilpotent, there exists some integer $n>0$ so that

$$
F_{1} F_{2} \cdots F_{n}=0
$$

Let $N$ be the maximal element in $C_{n+1}$.

Let $S$ be the set of all sequences $\delta=\left(d_{1}, d_{2}, \ldots\right)$ where $d_{i} \in B_{i}$, and note that $\alpha \in S$. We will prove that for any element $\delta \in S$ we have $d_{1} d_{2} \cdots d_{N}=0$. To do so, we need additional notation. Given $\delta=\left(d_{1}, d_{2}, \ldots\right) \in S$ we define the active subsequence $\delta^{\prime}=\left(d_{1}^{\prime}, d_{2}^{\prime}, \ldots\right)$ by setting $d_{1}^{\prime}=d_{1}$ and for $i>1$ putting $d_{i}^{\prime}=d_{\varphi\left(d_{i-1}^{\prime}\right)}$. The fact that $\varphi\left(b_{i, j}\right)>i$ implies that this really is a subsequence.

Given a sequence $\delta$, with active subsequence $\delta^{\prime}$ as written above, let $f_{i}^{\prime}(x)=$ $f_{\varphi\left(d_{i-1}^{\prime}\right)}(x)$ denote the polynomial with $d_{i}^{\prime}$ occurring as a coefficient. Write

$$
d_{1} d_{2} \cdots d_{N}=d_{1}^{\prime} r_{1} d_{2}^{\prime} r_{2} \cdots d_{n}^{\prime} r_{n} r
$$


by combining terms as necessary. Note that $d_{i}^{\prime} r_{i}$ is a coefficient in $f_{i}^{\prime}(x) r_{i} \in F_{i}$. Hence $d_{1}^{\prime} r_{1} d_{2}^{\prime} r_{2} \cdots d_{n}^{\prime} r_{n} r$ is a term in the product $\prod_{i=1}^{n} f_{i}^{\prime}(x) r_{i}$, say at degree $k$. This product of polynomials is zero by equation (44). Hence

$$
d_{1}^{\prime} r_{1} d_{2}^{\prime} r_{2} \cdots d_{n}^{\prime} r_{n} r=-\sum_{\left(e_{1}^{\prime}, e_{2}^{\prime}, \ldots, e_{n}^{\prime}\right)} e_{1}^{\prime} r_{1} e_{2}^{\prime} r_{2} \cdots e_{n}^{\prime} r_{n} r
$$

where the sum is over all $n$-tuples where $e_{i}^{\prime}$ is a coefficient of $f_{i}^{\prime}(x)$, the product $e_{1}^{\prime} \cdots e_{n}^{\prime}$ occurs at degree $k$, and $\left(e_{1}^{\prime}, e_{2}^{\prime}, \ldots, e_{n}^{\prime}\right) \neq\left(d_{1}^{\prime}, d_{2}^{\prime}, \ldots, d_{n}^{\prime}\right)$. We let $\mathcal{E}_{\delta}=$ $\left\{\eta=\left(e_{1}, e_{2}, \ldots\right) \in S\right\}$ be the set of all sequences $\eta$ that are obtained from $\delta$ by the following process: replace the first $n$ terms of the active subsequence of $\delta$ with one of the sequences $\left(e_{1}^{\prime}, e_{2}^{\prime}, \ldots, e_{n}^{\prime}\right)$ satisfying the conditions above. In particular, we have the equality

$$
d_{1} d_{2} d_{3} \cdots d_{N}=-\sum_{\eta \in \mathcal{E}_{\delta}} e_{1} e_{2} e_{3} \cdots e_{N}
$$

Note that at least one of the $e_{i}^{\prime}$ (for each $\eta$ ) occurs in a higher degree than where $d_{i}^{\prime}$ occurs, unless the sum is over the empty set and hence equals 0 .

Given two sequences $\delta, \eta \in S$ we say that one can make a "valid move" from $\delta$ to $\eta$, written $\delta \mapsto \eta$, if $\eta \in \mathcal{E}_{\delta}$. Equation (5) says that any sequence in $S$ is a linear combination of sequences arrived at by valid moves. Valid moves can only change the first $N$ entries of a sequence. In particular, either there is a cycle of valid moves $\delta_{1} \mapsto \delta_{2} \mapsto \cdots \mapsto \delta_{p}=\delta_{1}$ with $p>1$, or after a fixed number of valid moves every sequence in $S$ is sent to zero. If the second case holds, then our theorem is established, for the product $a_{1} a_{2} \cdots a_{N}$ can be replaced by a linear combination of products arising from valid moves applied to $\alpha$, and this process can be repeated, eventually terminating in a linear combination of zero products.

So, suppose by way of contradiction that there is a sequence of valid moves $\delta_{1} \mapsto \delta_{2} \mapsto \cdots \mapsto \delta_{p}=\delta_{1}$ with $p>1$. We can define an ordering on active subsequences by lexicographical order. In other words $\left(d_{1}^{\prime}, d_{2}^{\prime}, \ldots\right)<\left(e_{1}^{\prime}, e_{2}^{\prime}, \ldots\right)$ if and only if there is an integer $m \geq 1$ so that $d_{i}^{\prime}=e_{i}^{\prime}$ when $i<m$ but $d_{m}^{\prime}$ occurs as the coefficient of a smaller power of $x$ than $e_{m}^{\prime}$ does. After renumbering our loop, we may suppose that $\delta_{1}$ has a maximal active subsequence. For each $\ell>0$ write the active subsequence of $\delta_{\ell}$ as $\delta_{\ell}^{\prime}=\left(d_{\ell, 1}^{\prime}, d_{\ell, 2}^{\prime}, \ldots\right)$. When passing from $\delta_{1}$ to $\delta_{2}$, only the active subsequence changes. Further, one of the entries must change to a coefficient of higher degree, as mentioned immediately after equation (5), say $d_{1, k_{0}}^{\prime}$ increases to $d_{2, k_{0}}^{\prime}$, with $k_{0}$ minimal. But since we are in a cycle of valid moves, this coefficient must later change again. This can only happen, due to the injectivity of $\varphi$, if some later active subsequence begins with $\left(d_{1,1}^{\prime}, d_{1,2}^{\prime}, \ldots, d_{1, k_{0}-1}^{\prime}\right.$, $\left.d_{2, k_{0}}^{\prime}\right)$, contradicting the maximality of $\delta_{1}$. Thus, there can be no cycles, and the theorem is proven.

Definition 19. Let $I$ be an indexing set, and for each $i \in I$ let $J_{i}$ be a subset of $R$. We say that the collection $\left\{J_{i}\right\}_{i \in I}$ is right vanishing if for each sequence $i_{1}, i_{2}, i_{3}, \ldots$ of distinct elements of $I$ and each sequence of elements $a_{i_{j}} \in J_{i_{j}}$, there exists an integer $n \geq 1$ so that $a_{i_{1}} a_{i_{2}} \cdots a_{i_{n}}=0$.

This looks similar to the definition of left $T$-nilpotence, except that we are not allowed to repeat an index and the elements are not restricted to a single set. However, we still have: 
Proposition 20. Let $R$ be a ring, and let $I$ be an indexing set. If $\left\{J_{i}\right\}_{i \in I}$ is a right vanishing set of left (or right) ideals, then $\left\{J_{i}[x]\right\}_{i \in I}$ is right vanishing. On the other hand, if $\left\{K_{i}\right\}_{i \in I}$ is a right vanishing set of left (or right) ideals in $R[x]$, then $\left\{A_{K_{i}}\right\}_{i \in I}$ is right vanishing.

Proof. The first statement is a consequence of König's tree lemma. The second follows from the proof of the previous theorem.

We thank the referee for pointing out the following application of Theorem 18 Given a ring $R$ and an indexing set $I$, we let $R F M_{I}(R)$ denote the ring of rowfinite matrices with rows and columns indexed by $I$. As proved by Patterson 8 , one has the equality $\operatorname{rad}\left(R F M_{I}(R)\right)=R F M_{I}(\operatorname{rad}(R))$ if and only if $\operatorname{rad}(R)$ is left $T$-nilpotent. In fact, as proved by Sexauer and Warnock [11, and subsequently generalized by Zelmanowitz [14, we have $r \in \operatorname{rad}\left(R F M_{I}(R)\right)$ if and only if $r \in$ $R F M_{I}(\operatorname{rad}(R))$ and the column left ideals of $r$ are right vanishing, where the column left ideals of $r=\left(r_{i j}\right)_{i, j \in I}$ are of the form

$$
B_{j}(r)=\left\{\sum_{i \in I} k_{i} r_{i j} \mid k_{i} \in R \text { and } k_{i}=0 \text { for almost all } i \in I\right\} .
$$

Putting this all together, we have:

Corollary 21. The Jacobson radical of $R F M_{I}(R[x])$ is the set of matrices $r \in$ $R F M_{I}(N[x])$, where $N=\operatorname{rad}(R[x]) \cap R$ is a nil ideal and the coefficient ideals for each of the column left ideals of $r$ are right vanishing.

\section{LOOSE ENDS}

Proposition 10 leaves open the possibility that the condition " $\sigma$ is an epimorphism of $R$ " could be weakened to " $\sigma$ is an endomorphism of $R$ ". But the following example shows this will not work, even for a monomorphism.

Example 22. We refer the reader to [7, Example 3.7]. Let $R$ be the subset of the $\mathbb{Z}_{>0} \times \mathbb{Z}_{>0}$ matrices over a field $K$ defined as follows

$$
R=\left\{M \mid M=\sum_{i, j=1}^{n} a_{i j} e_{i j}+a \sum_{i=n+1}^{\infty} e_{i i} \text { for some } n \in \mathbb{Z}_{>0} \text { and } a_{i j}, a \in K\right\},
$$

where $\left\{e_{i j}\right\}_{i, j \in \mathbb{N}}$ denotes the set of matrix units. In other words, $R$ is the set of infinite matrices with finite support, plus $K$-multiples of the identity matrix. The ring $R$ is a prime ring. Define a map $\sigma: R \rightarrow R$ by

$$
\sigma\left(\sum_{i, j=1}^{n} a_{i j} e_{i j}+a \sum_{i=n+1}^{\infty} e_{i i}\right)=a e_{11}+\sum_{i, j=1}^{n} a_{i j} e_{i+1, j+1}+a \sum_{i=n+2}^{\infty} e_{i i} .
$$

In other words,

$$
\left(\begin{array}{ccccc}
A & 0 & 0 & 0 & \ldots \\
0 & a & 0 & 0 & \ldots \\
0 & 0 & a & 0 & \ldots \\
\vdots & \vdots & \ddots & \ddots & \ddots
\end{array}\right) \mapsto\left(\begin{array}{ccccc}
a & 0 & 0 & 0 & \ldots \\
0 & A & 0 & 0 & \ldots \\
0 & 0 & a & 0 & \ldots \\
\vdots & \vdots & \ddots & \ddots & \ddots
\end{array}\right)
$$

where the 0 entries have the appropriate sizes. The map $\sigma$ is an injective ring endomorphism of $R$. Notice that $e_{11} \sigma(R)=K e_{11}$. Therefore, for any integer 
$k \geq 0$, we have $e_{11} x R x^{k} e_{11}=K e_{11} e_{2+k, 2+k} x^{k+1}=0$, and so $e_{11} x R[x ; \sigma] e_{11}=0$. But $e_{11} R \sigma\left(e_{11}\right) R$ is not nilpotent, as it contains $e_{11}=e_{11} e_{12} e_{22} e_{21}$.

Theorems 12 and 14 classify which properties pass up, or go down, in polynomial rings. One can ask the same question for finitely generated right ideals, and the result is much nicer.

Theorem 23. Let $R$ be a ring, and let $\mathcal{P}$ be a property in diagram (3). Let $J$ be a finitely generated right ideal of $R$, and let $I$ be a finitely generated right ideal of $R[x]$. If $J$ has property $\mathcal{P}$, then $J[x]$ necessarily has property $\mathcal{P}$, except in the case where $\mathcal{P}=$ "nil". If I has property $\mathcal{P}$, then so does $A_{I}$. No other implications among these properties exist, going up or down, except those implied by transitivity.

Proof. All claimed implications follow from Theorems 12 and 14 and Proposition 16. Nonexistence of additional implications follows from our earlier examples.

\section{ACKNOWLEDGEMENTS}

The second, third, and fourth authors are grateful for the kind hospitality they received from the Department of Mathematics at the University of Iowa in 2007. The third author was supported by the LG Yonam Foundation in the program year 2006. The fourth author was supported by a Korea Research Foundation Grant funded by the Korean government (KRF-2008-521-C00003). The fifth author was partially supported by the University of Iowa's Department of Mathematics, NSF VIGRE grant DMS-0602242. We also thank the referee for suggesting simplifications, references, and immediate applications.

\section{REFERENCES}

1. D. E. Fields, Zero divisors and nilpotent elements in power series rings, Proc. Amer. Math. Soc. 27 (1971), 427-433. MR0271100 (42:5983)

2. B. J. Gardner, Some aspects of T-nilpotence, Pacific J. Math. 53 (1974), 117-130. MR0360667 (50:13114)

3. B. J. Gardner and R. Wiegandt, Radical theory of rings, Monographs and Textbooks in Pure and Applied Mathematics, vol. 261, Marcel Dekker Inc., New York, 2004. MR 2015465 (2004m:16031)

4. A. A. Klein, Rings of bounded index, Comm. Algebra 12 (1984), no. 1-2, 9-21. MR.732182 (85c:16016)

5. The sum of nil one-sided ideals of bounded index of a ring, Israel J. Math. 88 (1994), no. 1-3, 25-30. MR.1303489 (96b:16016)

6. T. Y. Lam, A first course in noncommutative rings, second ed., Graduate Texts in Mathematics, vol. 131, Springer-Verlag, New York, 2001. MR.1838439(2002c:16001)

7. A. Leroy and J. Matczuk, Goldie conditions for Ore extensions over semiprime rings, Algebr. Represent. Theory 8 (2005), no. 5, 679-688. MR.2189578 (2006h:16048)

8. E. M. Patterson, On the radicals of rings of row-finite matrices, Proc. Roy. Soc. Edinburgh Sect. A 66 (1961/1962), 42-46. MR0142582(26:151)

9. E. R. Puczyłowski, Nil ideals of power series rings, J. Austral. Math. Soc. Ser. A 34 (1983), no. 3, 287-292. MR695913 (84d:16004)

10. E. R. Puczyłowski and A. Smoktunowicz, The nil radical of power series rings, Israel J. Math. 110 (1999), 317-324. MR1750432 (2001e:16027)

11. N. E. Sexauer and J. E. Warnock, The radical of the row-finite matrices over an arbitrary ring, Trans. Amer. Math. Soc. 139 (1969), 287-295. MR0238889 (39:249)

12. A. Smoktunowicz, Amitsur's conjecture on polynomial rings in $n$ commuting indeterminates, Math. Proc. R. Ir. Acad. 102A (2002), no. 2, 205-213. MR.1961638 (2004c:16046) 
13. A. Smoktunowicz and E. R. Puczyłowski, A polynomial ring that is Jacobson radical and not nil, Israel J. Math. 124 (2001), 317-325. MR.1856524 (2002f:16049)

14. J. M. Zelmanowitz, Radical endomorphisms of decomposable modules, J. Algebra 279 (2004), no. 1, 135-146. MR2078391 (2005e:16051)

Department of Mathematics, University of Iowa, Iowa City, Iowa 52242

E-mail address: camillo@math.uiowa.edu

Department of Mathematics and Research Institute for Basic Sciences, Kyung Hee University, Seoul 131-701, Republic of Korea

E-mail address: hcy@khu.ac.kr

College of Liberal Arts, Hanbat National University, Daejon 305-719, Republic of Korea

E-mail address: nkkim@hanbat.ac.kr

Department of Mathematics Education, Pusan National University, Pusan 609-735, REPUBLIC OF Korea

E-mail address: ylee@pusan.ac.kr

Department of Mathematics, Brigham Young University, Provo, Utah 84602

E-mail address: pace@math.byu.edu 